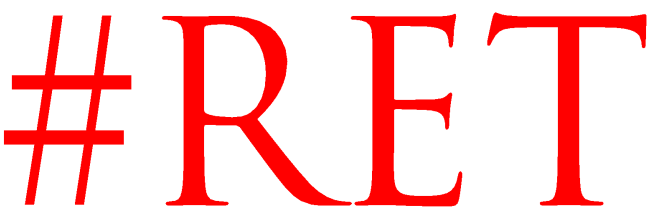

Revista Española de la Transparencia

Núm. 12. Primer Semestre. Enero-Junio de 2021, pp. 145-166

ISSN 2444-2607. Www.revistatransparencia.com

DOI: https://doi.org/10.51915/ret.110

CASOS Y SISTEMAS COMPARADOS

\title{
Breve análisis comparativo entre la regulación del Consejo para la Transparencia de Chile y el Consejo de Transparencia y Buen Gobierno de España
}

\author{
Jorge Astudillo Muñoz ${ }^{1}$ \\ Universidad Andres Bello (UNAB) \\ Chile
}

ORCID: 0000-0001-6562-1930

RECIBIDO: 27 de mayo de 2020

ACEPTADO: 9 de marzo de 2021

\begin{abstract}
RESUMEN: Este trabajo tiene como finalidad ofrecer un sucinto repaso de las principales características estructurales y funcionales del Consejo para la Transparencia chileno y luego contrastarlas con la regulación que hace del Consejo de Transparencia y Buen Gobierno la Ley 19/2013 en España. Lo que se busca es destacar las principales fortalezas y debilidades de la regulación chilena en relación a la normativa contenida en la Ley 19/2013 y con ello establecer algunas bases mínimas que permita abordar ambas legislaciones en forma comparada, procurando destacar los aspectos mejor logrados en cada una de ellas.
\end{abstract}

PALABRAS CLAVE: Acceso a la información pública, Publicidad activa, Consejo para la Transparencia, Consejo de Transparencia y Buen Gobierno.

1 Doctor en Derecho por la Universidad de Salamanca. Abogado. Licenciado en Ciencias Juridicas por la Universidad de Valparaíso (Chile). Master Oficial por la Universidad de Salamanca. Magister en Derecho con mención en Derecho Administrativo por la Pontificia Universidad Católica de Valparaíso (Chile). Profesor Regular de la Facultad de Derecho de la Universidad Andres Bello (UNAB). Sus líneas de investigación se centran principalmente en la transparencia, el acceso a la información pública, el combate a la corrupción y a la democracia. 
CONTENIDOS: 1.- Introducción. - 2. El Consejo para la Transparencia en Chile regulado por la Ley $N^{\circ}$ 20.285.- 3.-Breve análisis comparativo de la regulación del Consejo para la Transparencia chileno a la luz de la regulación española del Consejo de Transparencia y Buen Gobierno. 4.- Conclusiones.- Bibliografia.

\section{Brief comparative analysis between the regulation of the Council for Transparency (Chile) and the Council of Transparency and Good Governance (Spain)}

ABSTRACT: The purpose of this work is to offer a brief overview of the main structural and functional characteristics of the Chilean Council for Transparency and then contrast them with the regulations of the 19/2013 Transparency and Good Governance Council in Spain. What is sought is to highlight the main strengths and weaknesses of the Chilean regulation in relation to the regulations contained in Law 19/2013 and thereby establish some minimum bases that allow both laws to be addressed in a comparative manner, trying to highlight the aspects best achieved in each of them.

KEYWORDS: Access to public information, active publicity, Council for Transparency, Council for Transparency and Good Governance. 


\section{Introducción}

La existencia de un órgano autónomo e independiente encargado de velar por el cumplimiento de la normativa sobre publicidad activa y el derecho de acceso a la información pública constituyen una exigencia que resulta fundamental para el éxito de este tipo de legislaciones. Sin un ente dotado de estas características el derecho de acceso a la información pública terminaria irremediablemente siendo avasallado por intereses político-partidistas del gobierno de turno o por cualquier otro actor relevante dentro del sistema político.

La Ley Modelo Interamericana sobre Acceso a la Información Pública, por ejemplo, propone la creación de una Comisión de Información que estará encargada de "la promoción de la efectiva implementación de esta ley". Además, en esta propuesta de modelo legislativo se indica que esta Comisión "deberá tener personalidad jurídica completa, incluyendo para adquirir y disponer de propiedad y el poder de demandar y ser demandada" sin perjuicio de reconocerle "autonomía operativa de presupuesto y de decisión" y corresponderá al órgano legislativo "aprobar este presupuesto, el que deberá ser suficiente para que la Comisión de Información pueda cumplir con sus facultades adecuadamente".

El Convenio del Consejo de Europa para Acceso a los Documentos Públicos en su artículo $8^{\circ}$ dispone que "un solicitante que pida un documento oficial y cuyo acceso le ha sido denegado expresamente o implícitamente, parcialmente o por completo, tendrá acceso a un procedimiento de reclamación antes de la apelación ante un Tribunal u otra institución independiente e imparcial establecida por la ley".

La Relatoria Especial para la Libertad de Expresión de la OEA ha sostenido en forma reiterada que "para satisfacer plenamente las necesidades de acceso a la información de la sociedad y generar a largo plazo una cultura de la transparencia, los Estados no solo deben asegurar recursos sencillos, expeditos y gratuitos para controvertir las negativas de acceso a la información, también deben implementar adecuadamente las normas en materia de acceso a la información" y en este contexto, sin lugar a dudas, la existencia de un órgano dotado de competencias suficientes para fiscalizar el cumplimiento de esta normativa y garantizar el derecho de acceso es un imperativo ineludible.

En definitiva, de nada o de muy poco serviría reconocer en un sistema políticojurídico la publicidad activa y el derecho de acceso a la información pública si las personas no pueden impetrar su tutela ante órganos independientes e imparciales dotados de la facultad de imperio que le permita la ejecución de sus decisiones. Resaltamos la idea de la independencia y autonomía de la que debe estar dotado este tipo de órgano, pero ésta no solo debe ser funcional también debe ser 
presupuestaria. Esta autonomía e independencia debe ser reforzada por los principios de inamovilidad y por la imposibilidad de volver a designar a los integrantes de dicho órgano para uno o más períodos. Otra cuestión esencial dice relación con el nombramiento de las personas que ejercerán estas funciones. Consideramos que estos integrantes deben ser designados en el marco de un proceso transparente, donde intervengan los diversos poderes del Estado, previa consulta a organizaciones civiles y ciudadanas. Además, en estas designaciones deben primar criterios técnicos y no políticos.

Los diversos sistemas jurídicos, reconociendo la importancia de esta materia, configuran sus propias soluciones. Así por ejemplo, en el Reino Unido el Comisionado para la Protección de Datos se transformó en el Comisionado para la Información (Freedom of information act 2000, Part I, $N^{\circ} 18$ : the information commissioner and the information tribunal). Una solución similar se adoptó en Alemania en donde las funciones relacionadas con el derecho de acceso a la información regulado en la Ley de Libertad de Información del año 2005 se radican en la Comisión Federal de Protección de Datos Personales.

Por su parte, el artículo $17^{\circ}$ de la Ley Federal de Transparencia y Acceso a la Información Pública mexicana dispone que el Instituto Nacional de Transparencia, Acceso a la Información y Protección de Datos Personales (INAI) "es un organismo autónomo, especializado, independiente, imparcial y colegiado, con personalidad jurídica y patrimonio propio, con plena autonomía técnica, de gestión, capacidad para decidir sobre el ejercicio de su presupuesto y determinar su organización interna, responsable de garantizar en el ámbito federal, el ejercicio de los derechos de acceso a la información y la protección de datos personales, conforme a los principios y bases establecidos por el artículo 6o. de la Constitución, la Ley General, así como por lo previsto en esta Ley y demás disposiciones aplicables".

En Chile la Ley N² 20.285 de 20 de agosto de 2008 (Ley de Acceso a la Información Pública o LAIP) crea un órgano denominado Consejo para la Transparencia (CPT), mientras que en España la Ley 19/2013, de 9 de diciembre (LTAIBG) crea el Consejo de Transparencia y Buen Gobierno.

El presente trabajo tiene como finalidad hacer un repaso de las principales características estructurales y funcionales del Consejo para la Transparencia chileno y luego contrastarlas con la regulación que hace del Consejo de Transparencia y Buen Gobierno la Ley de Transparencia. Acceso a la Información Pública y Buen Gobierno en España. Lo que se busca es destacar las principales fortalezas y debilidades de la regulación chilena en relación a la normativa contenida en la Ley 19/2013 y proponer la posibilidad que algunas soluciones contenidas en ellas pueda ser replicada en forma reciproca. 
Con la finalidad de alcanzar los objetivos planteados en este artículo, en primer lugar, se lleva a cabo un análisis del Consejo para la Transparencia chileno a la luz de la regulación que hace la Ley $N^{\circ}$ 20.285. Este estudio abarca una breve referencia a su naturaleza jurídica y a sus aspectos órganicos y funcionales más relevantes. Posteriormente, y en base al estudio referido precedentemente, se ofrece un análisis comparativo entre la ley chilena y la Ley 19/2013 y su normativa relativa al Consejo de Transparencia y Buen Gobierno.

\section{El Consejo para la Transparencia de Chile (CPT)}

\subsection{Sobre su naturaleza jurídica}

El Consejo para la Transparencia, su organización y su ámbito competencial se encuentra regulado en el Título V de la LAIP, entre los artículos 31 a 44. De acuerdo a estos preceptos el Consejo para la Transparencia "es una corporación autónoma de Derecho Público, con personalidad jurídica y patrimonio propio, cuyo objeto es promover la transparencia de la función pública, fiscalizar el cumplimiento de las normas sobre transparencia y publicidad de la información de los órganos de la Administración del Estado y garantizar el derecho de acceso a la información".

La LAIP, en consecuencia, indica que el Consejo para la Transparencia "se establece como una corporación autónoma de Derecho Público, con personalidad jurídica y patrimonio propio y con potestad sancionatoria" (Bermúdez, 2012: 343).

En cuanto a la naturaleza jurídica del Consejo para la Transparencia, sostiene Bermúdez (2012: 343), que a pesar de que originalmente se intentó desvincular este órgano de la Administración del Estado, el Tribunal Constitucional en una sentencia del año 2008 estimó lo contrario, señalando que este ente si es parte de la Administración Pública (STC 1051/2008).

En una postura distinta, Rajevic Mosler plantea que al analizar las normas de la Ley de Transparencia relativas al CPT, la primera idea que brota es pensar que estamos en presencia de un "órgano de naturaleza sui generis", pues existen una serie de cualidades de las que ha sido dotado este organismo que nos hacen muy difícil reconducirlo a las categorías de la Ley Orgánica Constitucional de Bases Generales de la Administración del Estado (LBGAE). Se trata de una corporación autónoma de Derecho Público, facultada para proponer al Presidente de la República sus propios estatutos, no adscribiéndose por tanto al marco organizatorio general que regula a los Ministerios y demás servicios públicos creados por la ley para el ejercicio de la función administrativa (2010: 233).

Reforzando lo explicado por el profesor Rajevic Mosler, vamos a advertir que el CPT se encuentra excluido del Título II de la LBGAE, el que con su denominación "Normas Especiales", establece disposiciones dedicadas a la organización y 
funcionamiento de determinados organismos públicos a los que la doctrina chilena califica como "Administración del Estado en sentido restringido" (Cordero, 2015: 205). De esta forma el artículo 21 de la LBGAE dispone que las disposiciones de dicho Título II solo serán aplicables a Ministerios, Intendencias, Gobernaciones y servicios públicos creados para el cumplimiento de la función administrativa. Excluyendo expresamente en su inciso segundo al Consejo para la Transparencia, órgano que se regirá por sus propios estatutos legales.

Por otro lado, el inciso final del artículo $2^{\circ}$ de la LAIP, excluye al CPT de la nómina de órganos de la Administración que se regirán por ella, señalando que los demás órganos del Estado (donde se incluye al CPT) se ajustarán a las disposiciones de sus respectivas leyes orgánicas que versen sobre asuntos relacionados con la transparencia y la publicidad, el derecho de acceso y los procedimientos para su ejercicio.

Otra razón para sostener que el CPT no es un órgano de la Administración del Estado, sostiene Cordero Vega (2015: 205), se explica en que de ser entendido éste como parte de la Administración del Estado, necesariamente debería ser considerado dentro de la Administración del Estado descentralizada, esto es, como un órgano que tiene personalidad jurídica, patrimonio propio y que se encuentran sometido al Gobierno en virtud de un vínculo de supervigilancia a través del Ministerio respectivo. Sin embargo, y a pesar de que no hay duda en que el CPT tiene personalidad jurídica y patrimonio propio, mal podría controlar al Gobierno en materias de transparencia si, al final del día, estuviese sujeto a la supervigilancia del Presidente. "Al revés, su rol es precisamente supervigilar al Gobierno. Por ello, al no establecer esta relación típica el legislador nos dice, inequivocamente, que está resguardando la autonomía de este organismo" (Cordero, 2015: 205).

En suma, no existe disposición alguna en la legislación chilena que califique al Consejo para la Transparencia como parte de la Administración del Estado. Además, si tomamos en consideración su configuración, esta tesis cobra mucha fuerza. "Sin embargo, no puede sostenerse que el Consejo para la Transparencia sea una especie de islote autárquico en nuestra institucionalidad. La Constitución establece que el ejercicio de la soberanía se realiza por el pueblo a través del plebiscito y de elecciones periódicas y, también, por las autoridades que esta Constitución establece, como el Gobierno, la Administración, el Congreso Nacional, el Poder Judicial, etc. El Consejo para la Transparencia no puede estar en un limbo, debe adscribirse a una de esas autoridades. De las disposiciones que hemos comentado se desprende implícitamente que el Consejo para la Transparencia es una de las entidades autónomas a que se refiere el artículo 65, inciso $4 \mathrm{~N}^{\circ} 2$ y $\mathrm{N}^{0} 3^{2}$

\footnotetext{
${ }^{2}$ Constitución Política de Chile. Artículo 65, inciso cuarto: "Corresponderá, asimismo, al Presidente de
} la República la iniciativa exclusiva para: $2^{\circ}$ Crear nuevos servicios públicos o empleos rentados, sean 
de la Constitución, y que en tal calidad integran la Administración Pública como uno de los órganos creados para el cumplimiento de la función administrativa" (Rajevic, 2010: 233).

Atendido lo explicado, estamos de acuerdo con el profesor Rajevic cuando sostiene que el Consejo para la Transparencia puede situarse dentro de lo que la doctrina española ha llamado "administraciones independientes" (2010: 231).

Si hacemos caso a la doctrina española especializada, podemos indicar que las diferentes administraciones independientes reconocen entre sí a lo menos tres características comunes, las que consideramos, de alguna manera están presentes en el Consejo para la Transparencia chileno. Pomed Sánchez (1993: 121), sostiene que estas administraciones presentan como rasgos comunes, que en primer lugar están llamadas a actuar sobre sectores especialmente sensibles de intervención pública; en segundo lugar, el propósito que se persigue encomendando a las citadas administraciones independientes dicha actuación no es en modo alguno el de proceder a la constitución de ordenamientos jurídicos sectoriales, lo que únicamente sucede en la ordenación del sistema financiero. Antes bien, se trata de asegurar el alejamiento de las instancias de intervención de la lucha política partidista. Que ello se consiga depende, en gran medida, de los propios contendientes en esta lucha; y en tercer lugar, debemos destacar el requisito constitucional de la objetividad de la Administración Pública, este modelo organizativo añade otra nota caracterizadora, el de su neutralidad. Se trata de alcanzar objetivos (la transparencia informativa, la seguridad nuclear, la estabilidad monetaria, el buen funcionamiento del mercado de valores, o la defensa de la intimidad frente al uso indebido de la informática) que afectan al conjunto de la sociedad y cuya proyección temporal se extiende más allá de la vida de los Gobiernos de turno.

En buenas cuentas, las administraciones independientes, en el contexto del Estado Social y su rol regulador en el panorama europeo, se caracterizan por reunir los siguientes rasgos: i) independencia o autonomía frente al electorado y las instituciones políticas configuradas por medio de la elección popular; ii) una dirección y un personal con credenciales técnicas y científicas sobre su ámbito material de actuación; y iii) poderes y potestades suficientes para ejercer sus competencias (Solanes, 2016: 95).

fiscales, semifiscales, autónomos o de las empresas del Estado; suprimirlos y determinar sus funciones o atribuciones; $3^{\circ}$ Contratar empréstitos o celebrar cualquier otra clase de operaciones que puedan comprometer el crédito o la responsabilidad financiera del Estado, de las entidades semifiscales, autónomas, de los gobiernos regionales o de las municipalidades, y condonar, reducir o modificar obligaciones, intereses $u$ otras cargas financieras de cualquier naturaleza establecidas a favor del Fisco o de los organismos o entidades referidos". 
El propio Rajevic (2010: 231-232), citando al administrativista Santamaría Pastor, recuerda que las administraciones independientes pueden ser de dos tipos. Están aquellas "entidades creadas para el desempeño de servicios de interés general o de funciones públicas relevantes, cuya fuerte incidencia en la dinámica política y en el régimen de libertades públicas exige su realización con arreglo a criterios de neutralidad y de profesionalidad, sin sesgos partidarios" y las "entidades creadas para la ordenación y disciplina de sectores económicos capitales (de agencias reguladoras se habla comúnmente, con una terminología tomada del lenguaje estadounidense) cuyo difícil equilibrio y fuerte incidencia en la vida económica general requieren asimismo una gestión neutral y profesional". En caso de asumir que el CPT es una de estas administraciones independientes, no hay duda que debe circunscribirse dentro del primer de los grupos mencionados.

Finalmente, y sin perjuicio de su naturaleza jurídica, hay un generalizado consenso en la sociedad chilena del acierto que significó la creación del Consejo para la Transparencia y la forma de cómo fue estructurado orgánica y funcionalmente para el éxito de la legislación sobre la transparencia creada a partir de la LAIP.

Sin embargo, este nuevo órgano del Estado no se puede sustraer de una importante crítica: según Valdivia Olivares, "por amplia aceptación de que goce hoy, este modelo de organización de la gestión pública enfrenta una crítica severa... las administraciones independientes chocan con el principio democrático" (2010: 74). En definitiva, estamos en presencia de un órgano que no depende del Presidente de la República, que tampoco tiene una autonomía constitucional, que ha sido investido de una importante facultad normativa y que, "con alcance sobre toda la administración presenta el riesgo de constituir una limitación a la dirección que el gobierno debe ejercer sobre la administración, proviniendo estas reglas de un órgano no electo y sin responsabilidad política" (Valdivia, 2010: 74).

\subsection{Organización y estructura del Consejo para la Transparencia}

De acuerdo al artículo 36 de la LAIP, "la dirección y administración superiores del Consejo corresponderán a un Consejo Directivo integrado por cuatro consejeros designados por el Presidente de la República, previo acuerdo del Senado, adoptado por los dos tercios de sus miembros en ejercicio".

La misma disposición establece que "el Presidente hará la proposición en un solo acto y el Senado deberá pronunciarse respecto de la propuesta como una unidad". Una norma de esta naturaleza busca incentivar los acuerdos políticos en el Senado, pues al exigir que este se pronuncie sobre todos los nombres propuesto como unidad, imponen al Presidente de la República presentar consejeros que se encuentren vinculados a las diversas tendencias políticas presentes en el Senado 
en un momento determinado, y por otro lado, al ser presentados como eventuales candidatos al Consejo ciudadanos de distintas posturas, dentro del Senado necesariamente se buscará llegar a un acuerdo, transando en el nombramiento de personeros determinados a fin de que se materialice la designación de los propios.

Estos consejeros ostentarán el cargo durante seis años, pudiendo ser designados para un nuevo período. La nueva designación solamente es para un solo período más. Por otro lado, estos consejeros se renovarán por parcialidades cada tres años. Consideramos que si bien la reelección trae consigo la ventaja de una mayor especialización en la persona que oficia de consejero, debe siempre ser descartada en órganos de naturaleza contralora como es el Consejo para la Transparencia, pues la sola posibilidad de reelección puede influir en forma negativa en la independencia del ciudadano o ciudadana que ejerce el cargo.

El Consejo Directivo elegirá de entre uno de sus miembros a su Presidente, y en caso de no haber acuerdo, esta elección se realizará mediante sorteo. La presidencia del Consejo será rotativa por dieciocho meses y el consejero designado o elegido Presidente, no podrá serlo por el resto de su actual período como consejero.

La LAIP también establece una serie de incompatibilidades para ser designado consejero del Consejo para la Transparencia. De tal modo, no podrán ser designados como tales los diputados y senadores, los miembros del Tribunal Constitucional, los Ministros de la Corte Suprema, consejeros del Banco Central, el Fiscal Nacional del Ministerio Público, ni las personas que conforman el alto mando de las Fuerzas Armadas y de las Fuerzas de Orden y Seguridad Pública.

Además, la misma norma sostiene que los cargos de consejero son incompatibles con los de ministros de Estado, subsecretarios, intendentes y gobernadores; alcaldes y concejales; consejeros regionales; miembros del Escalafón Primario del Poder Judicial; secretario y relatores del Tribunal Constitucional; fiscales del Ministerio Público; miembros del Tribunal Calificador de Elecciones y su secretariorelator; miembros de los tribunales electorales regionales, sus suplentes y sus secretarios-relatores; miembros de los demás tribunales creados por ley; funcionarios de la Administración del Estado, y miembros de los órganos de dirección de los Partidos Políticos.

La LAIP también contempla las causales y forma de remoción de los consejeros. Los consejeros serán removidos por la Corte Suprema, a requerimiento del Presidente de la República, de la Cámara de Diputados mediante acuerdo adoptado por simple mayoría, o a petición de diez diputados. Las causales por las cuales se puede pedir la remoción de un consejero son la incapacidad, el mal comportamiento o la negligencia manifiesta en el ejercicio de sus funciones. La 
Corte Suprema conocerá del asunto en pleno especialmente convocado al efecto y para acordar la remoción deberá reunir el voto conforme de la mayoria de sus miembros en ejercicio.

Pero además de la remoción, se contemplan otras causales de cesación del cargo de consejero, a saber: i) expiración del plazo por el que fue designado; ii) renuncia ante el Presidente de la República; iii) postulación a un cargo de elección popular; e iv) incompatibilidad sobreviniente, circunstancia que será calificada por la mayoría de los consejeros con exclusión del afectado.

En el caso que uno o más de los consejeros tuviere que cesar en el ejercicio de sus funciones, procederá la designación de un nuevo consejero, mediante una proposición unipersonal del Presidente de la República, de acuerdo al mismo procedimiento previsto en el artículo 36, y este nombramiento será por el tiempo que faltaré al consejero que haya producido la vacante.

\subsection{El Consejo para la Transparencia. Aspectos funcionales}

De conformidad a la LAIP el Consejo para la Transparencia "tiene por objeto promover la transparencia de la función pública, fiscalizar el cumplimiento de las normas sobre transparencia y publicidad de la información de los órganos de la Administración del Estado, y garantizar el derecho de acceso a la información".

De acuerdo a la LAIP el CPT está llamado a cumplir tres tipos de funciones, por así decirlo, generales, a saber: i) una labor de promoción; ii) una labor de fiscalización, y iii) una labor de tutela.

Pues bien, en el ejercicio de esta labor de promoción ${ }^{3}$, el Consejo debe ser el órgano encargado de "iniciar" o "impulsar" un proceso para crear una cultura de la transparencia en la sociedad chilena, tomando la iniciativa para que ello se concrete. Por su parte la labor de fiscalización apunta a verificar que los actos, ya sea de los órganos del Estado o de algún particular, se ajusten a la normativa constitucional y legal vigente o bien a un sistema normativo de referencia. La fiscalización puede tener lugar en el ámbito público como en el privado. En relación con esta función fiscalizadora, al Consejo le corresponde realizar esta tarea respecto de los distintos órganos del Estado en relación con las obligaciones de publicidad y transparencia que les impone la LAIP. Finalmente, y he aquí uno de los principales aciertos de la ley de acceso chilena, es que se encomienda al Consejo garantizar a los individuos legitimados el acceso a la información para el evento que ésta no sea debida y oportunamente entregada por los órganos del

\footnotetext{
3 Según el Diccionario de la Lengua Española de la RAE (22 ${ }^{a}$ edición on-line), promover en su acepción $\mathrm{N}^{\circ} 1$ significa "iniciar o impulsar una cosa o un proceso, procurando su logro", y en su acepción $N^{\circ} 3$ "tomar la iniciativa para la realización o el logro de algo".
} 
Estado, ya sea bajo la modalidad de transparencia activa como en el evento que se desconozca el derecho de acceso propiamente tal.

Dentro del esquema general presentado por el artículo 32 de la LAIP, el artículo 33 del mismo cuerpo legal entrega al Consejo para la Transparencia las funciones y atribuciones ${ }^{4}$, que a continuación se mencionan: a) fiscalizar el cumplimiento de las disposiciones de esta ley y aplicar las sanciones en caso de infracción a ellas, b) resolver, fundadamente, los reclamos por denegación de acceso a la información que le sean formulados de conformidad a esta ley, c) promover la transparencia de la función pública, la publicidad de la información de los órganos de la Administración del Estado, y el derecho de acceso a la información, por cualquier medio de publicación; d) dictar instrucciones generales para el cumplimiento de la legislación sobre transparencia y acceso a la información por parte de los órganos de la Administración del Estado, y requerir a éstos para que ajusten sus procedimientos y sistemas de atención de público a dicha legislación; e) formular recomendaciones a los órganos de la Administración del Estado tendientes a perfeccionar la transparencia de su gestión y a facilitar el acceso a la información que posean; f) proponer al Presidente de la República y al Congreso Nacional, en su caso, las normas, instructivos y demás perfeccionamientos normativos para asegurar la transparencia y el acceso a la información; g) realizar, directamente o a través de terceros, actividades de capacitación de funcionarios públicos en materias de transparencia y acceso a la información; h) realizar actividades de difusión e información al público, sobre las materias de su competencia; i) efectuar estadísticas y reportes sobre transparencia y acceso a la información de los órganos de la Administración del Estado y sobre el cumplimiento de esta ley; j) velar por la debida reserva de los datos e informaciones que conforme a la Constitución y a la ley tengan carácter secreto o reservado; k) colaborar con y recibir cooperación de órganos públicos y personas jurídicas o naturales, nacionales o extranjeras, en el ámbito de su competencia; l) celebrar los demás actos y contratos necesarios para el cumplimiento de sus funciones; y, m) velar por el adecuado cumplimiento de la Ley $N^{\circ}$ 19.628, de protección de datos de carácter personal ${ }^{5}$, por parte de los órganos de la Administración del Estado.

\footnotetext{
${ }^{4}$ De acuerdo al Diccionario de la Lengua Española de la RAE (22 ${ }^{a}$ edición on-line), función en su acepción $\mathrm{N}^{\circ} 2$ es la "tarea que corresponde realizar a una institución o entidad, o a sus órganos o personas" y atribución en su acepción $\mathrm{N}^{\circ} 2$ se refiere a "cada una de las facultades o poderes que corresponde a cada parte de una organización pública o privada según las normas que las ordenen". Por lo tanto, podemos concluir que las atribuciones son en definitiva el conjunto de facultades de las que se encuentra dotado un órgano o una persona para cumplir las funciones que les han sido encomendadas.

${ }^{5}$ Publicada en el Diario Oficial No 36.451, de 28/08/1999.
} 
Consideramos que de la sola lectura de la norma transcrita en el párrafo precedente, la LAIP ha dotado al Consejo para la Transparencia de una serie de funciones y atribuciones que en principio lo transforman en un órgano importante y eficaz para asegurar el debido acceso de los ciudadanos a la información pública.

\section{Breve análisis comparativo de la regulación del Consejo de la Transparencia chileno a la luz de la regulación española del Consejo de Transparencia y Buen Gobierno}

La LAIP chilena encarga la tarea de garantizar el cumplimiento de su normativa y el derecho de acceso a la información pública a un órgano que se denomina Consejo para la Transparencia. La LTAIBG española encomienda esta función al Consejo de Transparencia y Buen Gobierno.

La forma en que han sido diseñados un órgano y otro, tanto en sus aspectos orgánicos como funcionales, es posiblemente una de las más marcadas e importantes diferencias entre el sistema de acceso a la información pública chileno y español.

En las páginas que sigue presentaremos sucintamente un análisis comparativo entre ambas normativas procurando destacar sus similitudes, fortalezas y debilidades.

\subsection{En cuanto a su estructura orgánica}

El Consejo para la Transparencia, órgano contemplado en la LAIP chilena en sus artículo 31 al 44, se define como "una corporación autónoma de Derecho Público, con personalidad jurídica y patrimonio propio, cuyo objeto es promover la transparencia de la función pública, fiscalizar el cumplimiento de las normas sobre transparencia y publicidad de la información y garantizar el derecho de acceso a la información pública".

Este Consejo para la Transparencia tiene una dirección y administración superior radicada en un Consejo Directivo integrado por cuatro consejeros designados por el Presidente de la República, previo acuerdo con el Senado, adoptado por las dos terceras partes de sus miembros en ejercicio. Estos consejeros ostentarán el cargo durante seis años, pudiendo ser designados para un nuevo período.

El Consejo de Transparencia y Buen Gobierno, se trata de un organismo público, previsto en la disposición adicional décima de la Ley 6/1997, de 14 de abril, de organización y funcionamiento de la Administración General del Estado, creado por la Ley 19/2013. Se trata de un órgano dotado de personalidad jurídica propia, con plena capacidad de obrar y que actúa con autonomía y plena independencia en el cumplimiento de sus fines. 
A diferencia de lo que sucede con el Consejo para la Transparencia chileno, el artículo 33.1 de la LTAIBG adscribe al Consejo de Transparencia y Buen Gobierno al Ministerio de Hacienda y Administraciones Públicas. En todo caso, esta adscripción no debería ser perjudicial en la medida que se respete realmente la autonomía que la ley le entrega al Consejo para el cumplimiento de sus fines.

Otro aspecto en que el Consejo de Transparencia y Buen Gobierno se aparta sustancialmente del modelo chileno es en su composición orgánica, ya que de acuerdo a la LTAIBG éste tiene una composición compleja pues se encuentra integrado por dos órganos, a saber: la Comisión de Transparencia y Buen Gobierno y el Presidente del Consejo, que además oficia como Presidente de la Comisión.

La Comisión a su vez estará compuesta por su Presidente (quien es el Presidente del Consejo, tal como lo hemos señalado previamente), un diputado, un senador, un representante del Tribunal de Cuentas, un representante del Defensor del Pueblo, un representante de la Agencia Española de Protección de Datos, un representante de la Secretaría de Estado de Administraciones Públicas y un representante de la Autoridad Independiente de Responsabilidad Fiscal.

En relación con la composición orgánica del Consejo de Transparencia y Buen Gobierno consideramos que esta conformación que incorpora al órgano actores relevantes de diversos órganos del Estado puede restar objetividad e independencia al Consejo, toda vez que en el ejercicio de sus funciones pudiesen primar en estos comisionados intereses corporativos más que criterios tendentes a una aplicación objetiva de la LTAIBG.

Por otro lado, estimamos que la aplicación de una normativa de esta naturaleza requiere de un grado de especialización y dedicación que es incompatible con la solución del artículo 36.3 de la LTAIBG que dispone que no será exigible a los integrantes de la Comisión una dedicación exclusiva a este oficio. Esta crítica también se extiende a los consejeros que integran el Consejo para la Transparencia chileno.

Donde si creemos que hay un importante acierto de la LTAIBG española, solución que debe ser adoptada en el modelo chileno, es la disposición contenida en el artículo 37 de esta norma en virtud de la cual se dispone que el Presidente del Consejo será nombrado por un periodo no renovable de cinco años. Debemos insistir en la idea de que la no reelección o redesignación incrementa en forma importante los niveles de independencia y autonomía, especialmente importante en un órgano de naturaleza contralora y garantista de derechos, en la medida que las decisiones adoptadas no tomarán como elemento de análisis una posible nueva designación. Valga aclarar, que si bien en el sistema chileno el Presidente del Consejo para la Transparencia dura 18 meses en el cargo y no puede volver a ser 
nombrado durante su período, la crítica que se formula al modelo chileno es más bien a la posibilidad de volver a designar a los miembros del Consejo para la Transparencia por otro período.

Finalmente respecto de la organización interna del Consejo de Transparencia y Buen Gobierno creemos que no resulta del todo acertada la decisión del legislador contenida en el artículo 39.2 de la LTAIBG de encargar al Consejo de Ministros aprobar mediante Real Decreto el Estatuto de Consejo, en donde se establecerá su organización, estructura y funcionamiento, así como todos los aspectos que sean necesarios para el cumplimiento de sus funciones. Si se reconoce al Consejo de Transparencia y Buen Gobierno personalidad jurídica y autonomía funcional, resulta negativo que se le prive de la facultad de dictar sus propios estatutos.

En el caso chileno, a la luz de lo dispuesto en el artículo 41 de la LAIP, los estatutos del Consejo para la Transparencia serán propuestos al Presidente de la República por el propio Consejo por, a lo menos una mayoría de tres cuartas partes de sus miembros, y su aprobación se dispondrá mediante decreto supremo expedido a través del Ministerio Secretaría General de la Presidencia.

Como es posible observar, la ley chilena reconoce un mayor grado de incidencia al Consejo de Transparencia que la LTAIBG al Consejo de Transparencia y Buen Gobierno, situación que puede redundar en el logro de una mayor o a lo menos más eficaz autonomía de uno u otro organismo.

\subsection{En cuanto a su estructura funcional}

En relación con los aspectos funcionales es posible constatar importantes diferencias entre la configuración del Consejo para la Transparencia chilena y el Consejo de Transparencia y Buen Gobierno de España.

De acuerdo a la normativa chilena el Consejo para la Transparencia se encuentra llamado a cumplir tres tipos de funciones, a saber: i) una función de promoción; ii) una función de fiscalización; y iv) una función de tutela o garantía.

En el ejercicio de la labor de promoción, el Consejo para la Transparencia se alza como el órgano encargado de impulsar un proceso que lleve a construir una cultura de la transparencia en la sociedad chilena y así mejorar los índices de probidad en el ejercicio de la función pública y disminuyendo de esta forma los actos de corrupción.

En el ejercicio de la función de fiscalización, corresponde al Consejo llevar a cabo las tareas que sean necesarias a fin de asegurar que los órganos del Estado sometidos a la LAIP cumplan con la normativa de publicidad activa y acceso a la información pública contenida en ella. 
El Consejo para la Transparencia también lleva a cabo una labor de tutela que le es encomendada por la LAIP, en virtud de la cual debe garantizar a los sujetos legitimados el acceso a la información pública para el evento que ésta no sea debida y oportunamente entregada por los órganos del Estado, ya sea bajo la modalidad de transparencia activa como en el evento que se desconozca el derecho de acceso propiamente tal.

Todas estas funciones se ven reforzadas por el innegable rol sancionador reconocido al Consejo para la Transparencia, tanto por la propia LAIP como por los tribunales de justicia 6 .

Desde la entrada en vigencia de la LAIP chilena le ha correspondido al Consejo para la Transparencia efectuar diversas funciones que la ley le encomienda dentro

6 El 27 de diciembre de 2016, la Corte Suprema rechazó un recurso de apelación deducido por el rector de la Universidad de Santiago (Usach), en contra de una sentencia previa de la Corte de Apelaciones de Santiago, que lo obligaba a pagar el 20\% de su remuneración mensual por infringir la Ley de Transparencia, tal como lo estableció una resolución del Consejo para la Transparencia. La Corte Suprema no solo ratificó en forma integra el fallo del tribunal de alzada, confirmando la multa hacia la autoridad universitaria, sino que además revalidó la potestad sancionatoria que tiene el Consejo para la Transparencia.

Los hechos que originaron la aplicación de la sanción se remontan al año 2013, a raíz de dos solicitudes de información realizadas por ex docentes de la USACH, a quienes se les suprimieron sus cargos a raíz de un proceso de reestructuración impulsado por el rector Zolezzi. Los académicos solicitaron a la casa de estudios los antecedentes que tuvo en consideración esa autoridad universitaria para decidir la supresión de sus cargos, los que no fueron entregados. Esto motivó que los solicitantes de información dedujeran ante el Consejo para la Transparencia, una serie amparos (roles C171-13, C573-13, C574-13, y C1151-13) que fueron acogidos dando la razón a los ex docentes. Sin embargo, la Universidad presentó tres reclamos de ilegalidad en contra de las decisiones de amparo del CPLT ante la Corte de Apelaciones de Santiago, los cuales fueron rechazados por el tribunal de alzada, concluyendo que las decisiones del CPLT se ajustan a derecho y que "la información requerida existe en poder de la universidad y debe ser proporcionada". En consecuencia, correspondía que la USACH entregara la información requerida, en la forma y dentro de los plazos dispuestos. No obstante, ello no fue cumplido, lo que fue denunciado por los ex docentes ante el CPLT. Tras ello, el CPLT solicitó el cumplimiento de lo resuelto por la Corte de Apelaciones, circunstancia que no se verificó por parte del rector, incurriendo con ello en un incumplimiento a las normas que regulan el derecho el derecho de acceso a la información pública. Con estos antecedentes, el Consejo Directivo del CPLT determinó solicitar a la Contraloría la instrucción de un sumario administrativo en contra del rector Zolezzi que concluyó con la multa del 20\% de su remuneración. Tras esto, la autoridad universitaria interpuso un recurso de protección lo que fue rechazado por la Corte de Apelaciones, ratificando que la potestad sancionatoria por incumplimientos a la Ley de Transparencia, radica únicamente en el CPLT y que es indelegable. Después de esa instancia, Zolezzi recurrió al máximo tribunal, que finalmente ayer, ratificó la sentencia apelada. Disponible en: http://www.cplt.cl/corte-suprema-ratifica-resolucion-del-consejo-para-latransparencia-que-sanciona-al-rector-de-la-usach/consejo/2016-12-28/140023.html (consulta 22/01/2017). 
de las cuales destaca la ya mencionada función de fiscalización del cumplimiento de sus disposiciones y sancionar a los funcionarios responsables de estas infracciones.

En el caso del Consejo de Transparencia y Buen Gobierno español el artículo 38 de la LTAIBG establece en sus dos apartados las funciones encomendadas tanto al Consejo como al Presidente de este organismo. Como se puede apreciar, este artículo 38 reconoce una serie de funciones que principalmente dicen relación con fines de asesoramiento, colaboración y promoción, pero no se vislumbran funciones decisorias relevantes, menos aún con carácter sancionatorio.

En el caso del Presidente del Consejo de Transparencia y Buen Gobierno, el artículo 38.2 letra c) le confiere competencia para resolver de una reclamación potestativa en relación con el artículo 24 de la LTAIBG, la que tiene condición sustitutiva de los recursos administrativos. En este sentido se vislumbra alguna competencia decisoria radicada en el Consejo, pero sin la facultad de aplicar sanciones, esta facultad es irrelevante.

Del análisis de la LTAIBG y especialmente del artículo 38 se desprende que el Consejo carece de facultades sancionatorias, situación que lo pone en una clara desventaja como órgano encargado de velar por el cumplimiento de su normativa.

\subsection{Mecanismos de reclamos ante la negativa injustificada de acceso y sanciones}

\section{a. Mecanismos de reclamo}

La configuración estructural y funcional de los órganos referidos en este trabajo no es una cuestión baladí toda vez que ella redunda en definitiva en la efectividad de los mecanismos de reclamo que se ponen a disposición de la ciudadanía en caso que la información pública solicitada sea negada sin que exista una causa legal que se encuentre efectivamente comprobada y las ulteriores sanciones para el evento que se incumpla la normativa.

Tanto en el caso de la LAIP chilena como la LTAIBG española hay un importante aporte a la transparencia, ya que en ninguno de estos sistemas de acceso a la información se ha establecido como vía de reclamación el agotamiento de la vía administrativa sino que se ha permitido el acceso directo a un órgano independiente del servicio público que ha denegado la información.

Tal como planeta Martín Delgado (2016: 377), a propósito de la LTAIBG española, en una apreciación que resulta plenamente aplicable al modelo chileno, esta decisión del legislador se encuentra plenamente justificada, tanto por razones de especialización en la materia como por motivos de independencia. Así, "la 
necesidad de optar por mecanismos sustitutivos de los recursos ordinarios resulta evidente en un sector, como el de transparencia, tan sensible a intereses políticos. Ha de tenerse presente que el acceso a la información pública, en el contexto de una Administración habituada a la opacidad, genera una inercia de rechazo a ofrecer la información requerida por el ciudadano, especialmente en la cúpside de la organización administrativa, que haría inoperante cualquier recurso administrativo tradicional, tanto el de reposición como particularmente el de alzada".

La LAIP chilena dispone que vencido el plazo para la entrega de la información requerida, o denegada la solicitud, el solicitante tendrá derecho a recurrir ante el Consejo para la Transparencia, solicitando el amparo de su derecho ${ }^{7}$.

Este reclamo debe señalar claramente cuál es la infracción cometida y los hechos que la configura, acompañando los medios de prueba que los acrediten. Debe presentarse dentro del plazo de 15 días, que se cuentan desde la notificación de la denegación del acceso o desde que haya expirado el plazo previsto para la entrega de la información.

Recibida esta reclamación el Consejo para la Transparencia notificará al órgano de la Administración que corresponda y al tercero involucrado si lo hubiere. Tanto la autoridad reclamada como el tercero involucrado podrán formular sus descargos dentro de los 10 días hábiles siguientes.

La resolución que falle el reclamo deducido se dictará dentro de quinto día hábil una vez vencido el plazo para formular descargos u observaciones. En esta misma resolución el Consejo podrá señalar la necesidad de iniciar un procedimiento disciplinario para establecer las responsabilidades que correspondan.

Por último la LAIP chilena dispone que en contra de la decisión del Consejo para la Transparencia procede un reclamo de ilegalidad ante la Corte de Apelaciones respectiva. La Corte de Apelaciones dictará sentencia dentro del término de 10 días contados desde la fecha en que se celebre la audiencia probatoria si la hubiere o desde que quede ejecutoriada la resolución que declara cerrado el término probatorio.

La LAIP señala categóricamente que contra esta resolución de la Corte de Apelaciones no procederá recurso alguno.

7 A noviembre de 2016, según reportes estadísticos del propio Consejo para la Transparencia han ingresado desde el año 2009 un total de 17.560 reclamos, de los cuales 11.238 (67\%) han sido formulados en contra de órganos centrales y 6.322 (36\%) en contra de municipalidades. Fuente: Reportes estadísticos Consejo para la Transparencia. Disponible en: http://extranet.consejotransparencia.cl/web_RSWV2/paginas/Verlnformacion.aspx (consulta 22/01/2017). 
Por último la LAIP dispone que para el evento de acogerse el reclamo de ilegalidad interpuesto en contra la denegación de acceso a la información, la sentencia señalará un plazo para la entrega de dicha información y en esta misma resolución la Corte de Apelaciones podrá señalar la necesidad de iniciar un procedimiento disciplinario para establecer si algún funcionario o autoridad han incurrido en algunas de las infracciones previstas en la ley.

La solución adoptada por la LTAIBG española difiere sustantivamente de la normativa chilena la que como acabamos de indicar entrega en primer término al Consejo para la Transparencia la competencia de conocer y resolver de los amparos que de deduzcan en contra de la negativa de entrega de la información y contempla la intervención de la justicia ordinaria solo por vía de reclamo de ilegalidad en contra de la decisión del Consejo.

En el caso de la ley española a la luz de lo dispuesto en el artículo 23 de la misma, la reclamación ante el Consejo de Transparencia y Buen Gobierno tiene un carácter facultativo y sustitutivo de los recursos administrativos. En otras palabras, el solicitante que no ha tenido satisfecha su pretensión de obtención de la información requerida puede interponer una reclamación ante el Consejo de Transparencia y Buen Gobierno o bien recurrir a la vía contenciosa administrativa.

Si el ciudadano puede optar entre la vía administrativa o bien recurrir al Consejo es posible deducir (aunque no lo diga expresamente la ley) que las resoluciones de éste tienen mérito ejecutivo. Dada la trascendencia de esta cuestión consideramos que el legislador debió consagrar este efecto de las resoluciones del Consejo en forma explícita.

La reclamación ante el Consejo se interpondrá en el plazo de un mes contado desde el día siguiente a la notificación a aquel en que se producen los efectos del silencio administrativo. Nótese que a diferencia de la ley chilena, la LTAIBG otorga un plazo sustancialmente mayor para recurrir ante el Consejo de Transparencia y Buen Gobierno.

La LTAIBG establece como plazo máximo para resolver y notificar las resoluciones que recaigan en los procedimientos de reclamación, tres meses, tras los cuales la reclamación se entenderá por desestimada en caso de no existir este pronunciamiento $u$ omitirse la notificación. Esta disposición consagrada en el artículo 24.4 de la LTAIBG nos merece dos comentarios. En primer lugar la forma en que se encuentra redactada la norma da pie a dejar en la indefensión al ciudadano requirente de la información, pues la norma solo exige el transcurso de término de tres meses para que la reclamación se entienda por desestimada, sin importar que esta inactividad no sea imputable al propio Consejo por ejemplo. $Y$ en segundo lugar, el plazo máximo de tres meses resulta excesivo a la luz de los intereses del 
solicitante que puede requerir la información en forma urgente, especialmente en comparación a la ley chilena la cual contempla un término para el fallo del amparo que no excede de los 15 días hábiles desde el momento en que es deducido.

Por último, valga recordar que de acuerdo a lo dispuesto en el artículo 23.1 de la LTAIBG la reclamación prevista en el artículo 24 se considerará sustitutiva de los recursos administrativos de conformidad a lo dispuesto en el artículo 107.2 de la Ley 30/1992, de 26 de noviembre.

Además, de acuerdo a lo dispuesto en el mismo artículo 23.2 en contra de las resoluciones dictadas por la Casa de la Majestad el Rey, el Congreso de los Diputados, el Senado, el Tribunal Constitucional y el Consejo General del Poder Judicial, Banco de España, Consejo de Estado, Defensor del Pueblo, Tribunal de Cuentas, Consejo Económico y Social y las instituciones autonómicas análogas en sus actividades sujetas al Derecho Administrativo, solo cabrá el recurso contencioso administrativo. La ley excluye expresamente la competencia de Transparencia y Buen Gobierno para conocer de la reclamación ante la negativa de entrega de la información según la LTAIBG.

\section{b. Sanciones}

La LAIP chilena establece el régimen de infracciones y sanciones en el Título VI de la misma. Así, la autoridad o jefatura superior del órgano o servicio de la Administración del Estado, que requerido en forma legal, hubiese denegado infundadamente el acceso a la información, será sancionado con una multa que oscila entre el $20 \%$ el 50\% de su remuneración. Del mismo modo, la no entrega oportuna de la información en la forma decretada, una vez que esta entrega fue ordenada por resolución firme, será sancionada con multa del 20\% al 50\% de la remuneración correspondiente.

En caso de que el funcionario sancionado persistiere en su actitud, se le aplicará el duplo de la sanción indicada precedentemente y la suspensión en el cargo por un lapso de cinco días.

Este mismo régimen sancionatorio se aplica en caso de incumplimiento injustificado de las normas sobre transparencia activa.

Por último, de acuerdo a la ley chilena, el órgano competente para aplicar estas sanciones es el propio Consejo para la Transparencia ${ }^{8}$. Tal como lo indicamos en el

8 En el periodo comprendido entre abril de 2009 a noviembre de 2016, 262 funcionarios de la Administración del Estado han sido sancionados por incumplimiento de las obligaciones contenidas en la Ley $N^{\circ}$ 20.285. De estos 262 funcionarios 3 corresponden a Jefe Superior de Servicio, 117 Alcaldes de municipalidades, 79 encargados o Directores de Departamento de Control , 29 encargados de transparencia,13 secretarios municipales, 12 administradores municipales y 9 funcionarios que desempeñan otro tipo de cargo. En total todas estas multas han significado un 
Capítulo destinado al estudio del Consejo para la Transparencia, este órgano tiene un incuestionado rol sancionador.

A diferencia de lo que sucede con la LAIP chilena, el Título I, sobre transparencia de la actividad pública de la LTAIBG española no contempla sanciones para el evento de incumplimiento de las normas sobre acceso a la información pública.

Solamente se dispone en el artículo 9.3, a propósito de la publicidad activa, que "el incumplimiento reiterado de las obligaciones de publicidad activa tendrá la consideración de infracción grave a los efectos de la aplicación a sus responsables del régimen disciplinario previstos en la correspondiente normativa reguladora".

Por su parte el artículo 20.6 incluido en la Sección $2^{a}$ del Capítulo Tercero que regula el ejercicio del derecho de acceso a la información pública, dispone que "el incumplimiento reiterado de la obligación de resolver en plazo tendrá la consideración de infracción grave a los efectos de la aplicación a sus responsables del régimen disciplinario previsto en la correspondiente normativa reguladora".

La Ley 19/2013 no establece directamente un sistema sancionatorio para el evento de incumplimiento de sus disposiciones, debiendo por tanto aplicarse las normas generales. Afirmamos de este modo que el régimen sancionador de la LTAIBG es débil, ya que carece de un título especifico regulador de éste, "con un cuadro de infracciones, sanciones, procedimientos y cuestiones complementarias, que contrasta con el régimen previsto para el Buen Gobierno en el Título II" (Atienza, 2013: 333).

\section{Conclusiones}

La LAIP chilena encarga la tarea de garantizar el cumplimiento de su normativa y el derecho de acceso a la información pública a un órgano que se denomina Consejo para la Transparencia. La LTAIBG española encomienda esta función al Consejo de Transparencia y Buen Gobierno.

La forma en que han sido diseñados un órgano y otro, tanto en sus aspectos orgánicos como funcionales, es posiblemente una de las más marcadas e

ingreso al patrimonio fiscal de aproximadamente \$101.764.000 lo que equivale alrededor de 144.240, 271 Euros. Consejo para la Transparencia, Unidad de Seguimiento de Decisiones y Sumarios de la Dirección de Fiscalización. Jurisprudencia Sancionatoria: Ley de Transparencia 2009-2014. Santiago: Consejo para la Transparencia, 2014. Disponible en:

http://www.educatransparencia.cl/sites/default/files/jurisprudencia_sancionatoria_cplt_o.pdf (consulta 17/01/2017).

Revista Española de la Transparencia. RET. ISSN 2444-2607.

Núm. 12. Primer Semestre. Enero-Junio de 2021, pp. 145-166

DOI: https://doi.org/10.51915/ret.110 
importantes diferencias entre el sistema de acceso a la información pública chileno y español.

La LAIP ha dotado al Consejo para la Transparencia de una serie de funciones y atribuciones que tiene el mérito de convertirlo en un actor relevante en el sistema de acceso a la información pública en Chile. El principal acierto de la ley chilena es que dota al CPT de funciones fiscalizadoras en relación con el cumplimiento de las obligaciones que ésta impone y sancionatorias lo que refuerza su rol de garante del cumplimiento de la transparencia activa y del derecho de acceso a la información pública.

Las características de las que está dotado el CPT en Chile no están presenten en la configuración que hace la Ley 19/2013. De la lectura de la LTAIBG y, particularmente de su artículo 38 se desprende que el Consejo de Transparencia y Buen Gobierno carece de facultades sancionatorias, situación que lo pone en una clara desventaja como órgano encargado de velar por el cumplimiento de la normativa contenida en la misma en materia de publicidad activa y acceso a la información pública.

\section{Bibliografía}

\section{Doctrina}

Bermúdez, J. 2012. Derecho administrativo general. Santiago: Legal Publishing Chile.

Consejo para la Transparencia, Unidad de Seguimiento de Decisiones y Sumarios de la Dirección de Fiscalización. Jurisprudencia Sancionatoria: Ley de Transparencia 2009-2014. Santiago: Consejo para la Transparencia, 2014.

Cordero Vega, L. 2015. Lecciones de derecho administrativo. Santiago de Chile: Legal Publishing Chile.

Martín Delgado, I. 2026. «La reclamación ante el Consejo de Transparencia y Buen Gobierno: un instrumento necesario, útil y ¿eficaz?», en López Ramón, F. (coordinador), Las vias administrativas de recurso a debate. Actas del XI Congreso de la Asociación Española de Profesores de Derecho Administrativo, Zaragoza, Instituto Nacional de Administración Pública, 369-431.

Navarro Atienza, M. Ley de Transparencia, Acceso a la Información Pública y Buen Gobierno y el Convenio del Consejo de Europa sobre acceso a los documentos públicos. En Administración de Andalucia: Revista Andaluza de Administración Pública, No 87, 2013, pp. 323-343. 
Pomed Sánchez, L. A. 1993. «Fundamento y naturaleza jurídica de las administraciones independientes», en Revista de Administración Pública (España), No 123, septiembre-diciembre: 117-169.

Rajevic Mosler, E. 2010. «El Consejo para la Transparencia como "administración independiente"», en Letelier, R y Rajevic, E. (coordinadores), Transparencia en la administración pública, Santiago de Chile, Legal Publishing Chile, 231-247.

Solanes Mullor, J. 2016. Administraciones independientes y Estado regulador. El impacto de la Unión Europea en el Derecho Público español. Madrid: Congreso de los Diputados.

Valdivia Olivares, J. 2010. «Lo que oculta la transparencia», en Letelier, R y Rajevic, E. (coordinadores), Transparencia en la administración pública, Santiago de Chile, Legal Publishing Chile, 69-78.

\section{Documentos y jurisprudencia}

Asamblea General de la Organización de Estados Américanos (OEA). 2010. Ley Modelo Interamericana sobre Acceso a la Información Pública. Resolución AG/Res. 2607 (XL-0/10), 4ta Sesión Plenaria de 8 de junio.

Consejo de Europa. 2009. Convenio del Consejo de Europa para Acceso a los Documentos Públicos, Tromsø, 18 de junio.

Relatoría Especial para la Libertad de Expresión. 2015. El derecho de acceso a la información pública en las Américas: entidades especializadas para la supervisión y cumplimiento: Informe temático contenido en el informe anual 2014. Washington D.C.,

Tribunal Constitucional Chileno. Sentencia 1051/2008 de 10 de julio de 2008. Control de constitucionalidad del proyecto de ley, aprobado por el Congreso Nacional, sobre acceso a la información pública. 